\title{
Skin of the Face
}

National Cancer Institute

\section{Source}

National Cancer Institute. Skin of the Face. NCI Thesaurus. Code C33561.

The integumentary covering of the face. 